\title{
Componentes alimentarios en la dieta del lobo de páramo Lycalopex culpaeus en la plataforma del aeropuerto Mariscal Sucre, parroquia Tababela, Cantón Quito, Pichincha, Ecuador
}

\author{
Dieta del lobo de páramo en la \\ plataforma del aeropuerto Mariscal Sucre
}

\author{
Darío Sebastián Reina Moreno
}

Investigador Independiente

Autor para Correspondencia/ Corresponding Author, e-mail: darius_antubis@hotmail.com

\section{Food components in the diet of the paramo wolf Lycalopex culpaeus on the platform of the Mariscal Sucre airport, Tababela parish, Quito Canton, Pichincha, Ecuador}

\begin{abstract}
I collected 117 faeces from which we evidenced 471 rests corresponding to the following groups: mammals, birds, reptiles, insects and some plants. Mammals were the most diverse group with 6 species from which the wild rabbit Sylvilagus brasiliensis was the most frequent species in the diet. On the other hand, the beatles were the most abundant group of the register species. The statistical analysis showed a significant frequency variation by months suggesting that Lycalopex culpaeus was able to modify its diet according to the availability of preys in the Tababela Airport platform.
\end{abstract}

Keywords: Feeding, food items, feces, Lycalopex, Tababela

\section{Resumen}

Se recolectaron 117 fecas de las que se obtuvieron restos correspondientes a mamíferos, aves, reptiles, insectos y algunos restos vegetales. Los mamíferos fueron el grupo más diverso con 6 especies, siendo el conejo silvestre Sylvilagus brasiliensis la especie más frecuente dentro de la dieta. En relación con los invertebrados, específicamente los escarabajos fueron el grupo más abundante en la dieta, $62 \%$ de las especies registradas. El análisis estadístico mostró a la variación mensual de la frecuencia de presas como significativa, sugiriendo que el lobo de páramo es capaz de modificar su dieta en relación a la disponibilidad de presas dentro de la plataforma del aeropuerto de Tababela, al norte de Ecuador.

Aceptado /

Accepted:

2017-05-08

Publicado en línea / Published online: 2019-04-19 


\section{INTRODUCCIÓN}

El Lobo de Páramo o Zorro Andino Lycalopex culpaeus es el segundo cánido más grande de Sudamérica, después del lobo de crin (Chrysocyon brachyurus) [1] y cánido más grande que habita en nuestro país [2]. En el Ecuador se distribuye a lo largo de la región andina, en los bosques templados y páramos de todo el país [2,3], entre los 2600 y 4500 m.s.n.m. En la actualidad esta especie está considerada como Vulnerable, teniendo a la pérdida de hábitat por el avance de la frontera agrícola y ganadera y el crecimiento de poblados, como la principal amenaza para su conservación en nuestro país [3]. La dieta de Lycalopex culpaeus ha sido uno de los aspectos más estudiados de su ecología [4]. Sin embargo, en nuestro país la información sigue siendo escasa, la cual se restringe a un estudio realizado en el Boque Protector Jerusalén, Provincia de Pichincha [5] en el cual se registró que la dieta estuvo compuesta por mamíferos, aves, reptiles, insectos y vegetales. En estudios realizados en Chile [6] y Argentina [7] se reporta a los lagomorfos y roedores como los componentes principales dentro de la dieta de Lycalopex culpaeus; además, se ha registrado que los frutos constituyen un elemento importante en la dieta [8]. Por otra parte, en el sur de Perú [9] se menciona que la dieta de Lycalopex culpaeus posee como componente principal a los vegetales, complementada por roedores y artrópodos. De la misma forma se ha registrado que la dieta de este cánido varía estacional y geográfica mente, así también se ha detectado que en ciertas zonas los roedores componen una parte importante de la dieta [10]. A pesar de que la mayoría de los ítems alimentarios consumidos por Lycalopex culpaeus son de origen animal, es una especie que consume una gran cantidad de frutas, siendo por esto responsable de la dispersión de varias especies de plantas [11]. Esta amplia gama de recursos que son utilizados por Lycalopex culpaeus dan a conocer la importancia que tiene este cánido en su hábitat ya sea como un controlador de poblaciones de vertebrados e invertebrados o como dispersor de semillas. El objetivo de este estudio fue identificar las especies presa que componen la dieta de lobo de páramo con la finalidad de aportar con nuevos datos sobre la alimentación de este mamífero en nuestro país.

\section{MATERIALES Y MÉTODOS}

El estudio se realizó durante los meses de abril a diciembre de 2012, exceptuando el mes de junio, en el sector correspondiente a la plataforma del nuevo aeropuerto "Mariscal Sucre". En la parroquia de Tababela, al nororiente de la provincia de Pichincha ubicado entre las coordenadas 0,1535 S / 78,3586 W y 0,1015 S / 78,3560 W entre los 2335 m y $2450 \mathrm{~m}$ de altitud. Dentro del área se pudieron registrar varios indicios de la presencia del lobo de páramo como huellas, marcas y fecas; además se obtuvieron avistamientos que fueron registros concretos de la presencia de este cánido dentro de la plataforma. En el sector se encuentran remanentes correspondientes al Bosque y Arbustal semideciduo del norte de los Valles [12]. La vegetación está caracterizada por especies suculentas, espinosas y crasulentas; como Mimosa quitensis, Acacia macracantha, Caesalpinia spinosa, especies de cactos como Opuntia soederstediana y O. cilindirica que son parte del paisaje junto con Agave americana y Fourcrae andina, además de otras formaciones de arbustos en donde pueden encontrarse ciertas especies de epífitas como Tilandsia recurvata [12]. El área de estudio consiste en una meseta plana rodeada por tres 
quebradas, del río Santa Rosa por la zona oriental, y las quebradas de los ríos Guambi y Guayllabamba por la zona occidental. (Fig. 1).

Figura 1. Área de Estudio, Plataforma del Aeropuerto Mariscal Sucre, Parroquia Tababela, Cantón Quito.

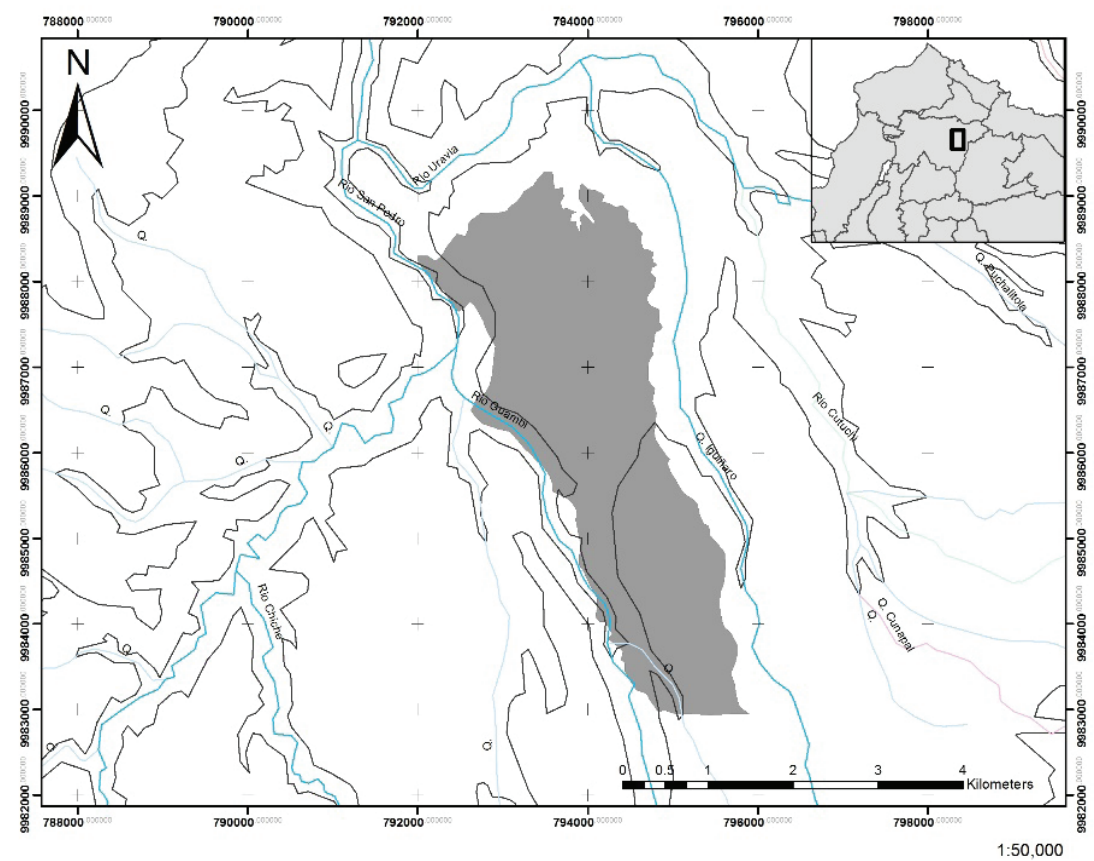

Se realizaron dos transectos de aproximadamente $8 \mathrm{~km}$ que cubrían las zonas occidental y oriental de la plataforma, los cuales se recorrieron mensualmente de manera simultánea, de igual forma se realizaron caminatas libres para cubrir la zona central de la plataforma.

Durante los recorridos se realizó la colecta de muestra fecales, a las mismas que se les asignó un código individual basado en las dos o tres primeras letras del mes en que fueron colectadas y seguidas por un numeral (por ejemplo: el código AB01, corresponde a la primera muestra fecal colectada durante el mes de abril). Las muestras fecales fueron secadas a temperatura ambiente $\left(24^{\circ} \mathrm{C}\right)$, y posterior a ello, se extrajeron los restos óseos, restos de invertebrados y material vegetal. Los restos óseos, garras y pelos fueron analizados en el laboratorio de la División de Mastozoología del Museo del Instituto Nacional de Biodiversidad, donde se realizó la identificación y el conteo de los individuos obtenidos mediante el uso de equipo de disección y un estéreo microscopio.

La representatividad dentro de la dieta para cada ítem alimentario fue establecida mediante la frecuencia de ocurrencia que consiste en el número de heces en el que se encuentra un ítem determinado dividido para el total de heces analizadas [6]. 
Para el análisis inferencial se utilizó el Programa PAST [13], donde se usó la prueba de G para evaluar la variación temporal de la frecuencia de presas en la dieta. El estudio corresponde al análisis de la dieta de L. culpaeus correspondiente a la época seca, por lo cual se resolvió evaluar la variación del uso de recursos dentro de una misma estación.

\section{RESULTADOS}

Dentro del estudio fueron analizadas 117 fecas, de las cuales se obtuvieron los restos de 9 especies, pertenecientes a 6 familias, 6 órdenes y 4 clases. La Clase Mammalia fue la más representativa con 6 especies correspondientes a 4 órdenes; seguido por la Clase Insecta con 2 especies y por último la Clase Reptilia con una especie. (Tabla 1).

Tabla 1. Frecuencia y Porcentaje de Ocurrencia de ítems alimentarios dentro de la Dieta de Lycalopex culpaeus en la Plataforma del nuevo Aeropuerto Mariscal Sucre, parroquia Tababela. Abreviaturas: $n f=$ Frecuencia de 0 currencia de las presas por mes; $\%$ = Porcentaje de la Frecuencia de 0currencia por mes en relación al porcentaje total de muestras analizadas.

\begin{tabular}{|c|c|c|c|c|c|c|c|c|c|c|c|c|c|c|c|c|}
\hline & \multicolumn{2}{|c|}{ Abril } & \multicolumn{2}{|c|}{ Mayo } & \multicolumn{2}{|c|}{ Julio } & \multicolumn{2}{|c|}{ Agosto } & \multicolumn{2}{|c|}{ Septiembre } & \multicolumn{2}{|c|}{ Octubre } & \multicolumn{2}{|c|}{ Noviembre } & \multicolumn{2}{|c|}{ Diciembre } \\
\hline $\begin{array}{l}\text { Número de } \\
\text { Muestras }\end{array}$ & \multicolumn{2}{|c|}{5} & \multicolumn{2}{|c|}{15} & \multicolumn{2}{|c|}{17} & \multicolumn{2}{|c|}{19} & \multicolumn{2}{|c|}{18} & \multicolumn{2}{|c|}{22} & \multicolumn{2}{|c|}{11} & \multicolumn{2}{|c|}{10} \\
\hline Item alimentario & $n_{f}$ & $\%$ & $n_{f}$ & $\%$ & $\mathbf{n}_{\mathbf{f}}$ & $\%$ & $n_{f}$ & $\%$ & $n_{f}$ & $\%$ & $n_{f}$ & $\%$ & $n_{f}$ & $\%$ & $\mathbf{n}_{\mathbf{f}}$ & $\%$ \\
\hline Barotheus andinus & 1 & 0,9 & 4 & 3,4 & 2 & 1,7 & 4 & 3,4 & 4 & 3,4 & 13 & 11,1 & 6 & 5,1 & 2 & 1,7 \\
\hline $\begin{array}{c}\text { Praogolopha } \\
\text { unicolor }\end{array}$ & 0 & 0,0 & 0 & 0,0 & 0 & 0,0 & 0 & 0,0 & 0 & 0,0 & 2 & 1,7 & 3 & 2,5 & 4 & 3,4 \\
\hline $\begin{array}{l}\text { Stenocercus } \\
\text { guentheri }\end{array}$ & 1 & 0,9 & 1 & 0,9 & 2 & 1,7 & 0 & 0,0 & 0 & 0,0 & 1 & 0,9 & 1 & 0,9 & 1 & 0,9 \\
\hline Akodon cf mollis. & 1 & 0,9 & 2 & 1,7 & 0 & 0 & 0 & 0,0 & 1 & 0,9 & 0 & 0,0 & 0 & 0,0 & 0 & 0,0 \\
\hline Phyllotis haggardi & 0 & 0,0 & 2 & 1,7 & 2 & 1,7 & 0 & 0,0 & 0 & 0,0 & 0 & 0,0 & 0 & 0,0 & 1 & 0,9 \\
\hline $\begin{array}{l}\text { Reithrodontomys } \\
\text { soderstromi }\end{array}$ & 1 & 0,9 & 2 & 1,7 & 4 & 3,4 & 1 & 0,9 & 2 & 1,7 & 4 & 3,4 & 1 & 0,9 & 0 & 0,0 \\
\hline $\begin{array}{l}\text { Sylvilagus } \\
\text { brasiliensis }\end{array}$ & 5 & 4,3 & 13 & 11,1 & 16 & 13,6 & 19 & 16,2 & 18 & 15,4 & 20 & 17 & 6 & 5,1 & 8 & 6,8 \\
\hline Didelphis pernigra & 0 & 0,0 & 0 & 0,0 & 1 & 0,9 & 0 & 0,0 & 0 & 0,0 & 0 & 0,0 & 0 & 0,0 & 0 & 0,0 \\
\hline $\begin{array}{l}\text { Conepatus } \\
\text { semistriatus }\end{array}$ & 0 & 0,0 & 0 & 0,0 & 0 & 0,0 & 1 & 0,9 & 0 & 0,0 & 0 & 0,0 & 2 & 1,7 & 0 & 0,0 \\
\hline Restos de Aves N/D & 0 & 0,0 & 0 & 0,0 & 4 & 3,4 & 2 & 1,7 & 1 & 0,9 & 2 & 1,7 & 0 & 0,0 & 0 & 0,0 \\
\hline Annona cherimola & 1 & 0,9 & 5 & 4,3 & 3 & 2,5 & 5 & 4,3 & 1 & 0,9 & 2 & 1,7 & 0 & 0,0 & 1 & 0,9 \\
\hline Zea mays & 0 & 0,0 & 0 & 0,0 & 0 & 0,0 & 0 & 0,0 & 1 & 0,9 & 0 & 0,0 & 0 & 0,0 & 0 & 0,0 \\
\hline $\begin{array}{c}\text { Restos Vegetales } \\
N / D\end{array}$ & 1 & 0,9 & 6 & 5,1 & 4 & 3,4 & 1 & 0,9 & 9 & 7,7 & 15 & 12,8 & 6 & 5,1 & 6 & 5,1 \\
\hline
\end{tabular}

De entre los mamíferos, el Orden Rodentia fue el más diverso, con tres especies (Reithrodontomys soderstromi; Phyllotis haggardi; Akodon cf. mollis), mientras que con una especie cada uno los órdenes Carnívora (Conepatus semistriatus), Didelphimorphia (Didelphis pernigra) y Lagomorpha (Sylvilagus brasiliensis), presentaron menor diversidad. 
En el caso de los insectos estuvieron representados por dos especies de escarabajos perteneciente a la Familia Melolonthidae, Barotheus andinus y Praogolopha unicolor.

Dentro de los reptiles en la dieta fue registrada la Guagsa, Stenocercus guentheri (Familia Tropiduridae), perteneciente al orden Sauria.

Por otra parte, dentro de la dieta se registró la presencia de semillas pertenecientes a la Chirimoya Annona cherimola y el Maiz Zea mays.

\section{Ocurrencia de presas}

Durante el análisis de las muestras fecales $(n=117)$ se registró a Sylvilagus brasiliensis como la presa más utilizada por L. culpaeus (89,7\%), seguido por: B. andinus $(30,8 \%)$ Reithrodontomys soderstromi (12,8\%), Phyllotis haggardi (4,3\%), Akodon cf. mollis (3,4\%).

En el caso de los mamíferos medianos, donde Conepatus semistriatus tuvo una frecuencia de ocurrencia del 2,6\% y Didelphis pernigra tuvo una frecuencia de ocurrencia del $0,9 \%$ dentro de la muestras analizadas.

Otro de los grupos importantes dentro de la dieta fueron los restos vegetales que mostraron una frecuencia de ocurrencia del 57,3\%; donde los restos vegetales no identificados fueron el ítem alimentario más frecuente, $41 \%$ de frecuencia de ocurrencia; de la misma forma dentro de este grupo se identificó a Annona cherimola, que presentó una ocurrencia del 15,4\% y por último se registró a Zea mays con una frecuencia de ocurrencia del $0,9 \%$ en las muestras analizadas.

Los insectos fueron el tercer grupo más frecuente en la dieta, siendo registrado en el $38,5 \%$ de la muestras analizadas, donde $B$. andinus fue el ítem alimentario más frecuente y seguido de Pragolopha unicolor el cual presentó una frecuencia de ocurrencia del 7,7\%.

Los reptiles dentro de la dieta, representados por Stenocercus guentheri, tuvieron una frecuencia del $6 \%$ dentro de la muestras, siendo uno de los grupos menos frecuentes en la dieta.

En el caso de las aves se encontraron en el 7,7\% de las muestras analizadas, sin embargo por el estado de las estructuras no se llegó a establecer una identificación taxonómica para este grupo.

\section{Variación Mensual en la Ocurrencia de Presas}

Durante los ochos meses de estudio fueron analizadas un total de 117 especies, con un promedio de 14,6 muestras (fecas) durante todo el tiempo de trabajo en campo; donde fueron los meses de octubre y agosto cuando se registró un mayor número de muestras (22 y 19, respectivamente), por el contrario, los meses de abril y diciembre fueron cuando se registró un menor número de muestras (5 y 10, respectivamente). La prueba de G utilizada para evaluar la variación mensual en el uso de presas mostró un resultado significativo ( $p=0.012$ ), mostrando la capacidad de L. culpaeus, para modificar su dieta en cortos periodos de tiempo, a fin de poder sobrevivir. (Fig. 2). 
Figura 2. Frecuencia de Ocurrencia de Presas de la Dieta de Lycalopex culpaeus en la plataforma de Tababela.

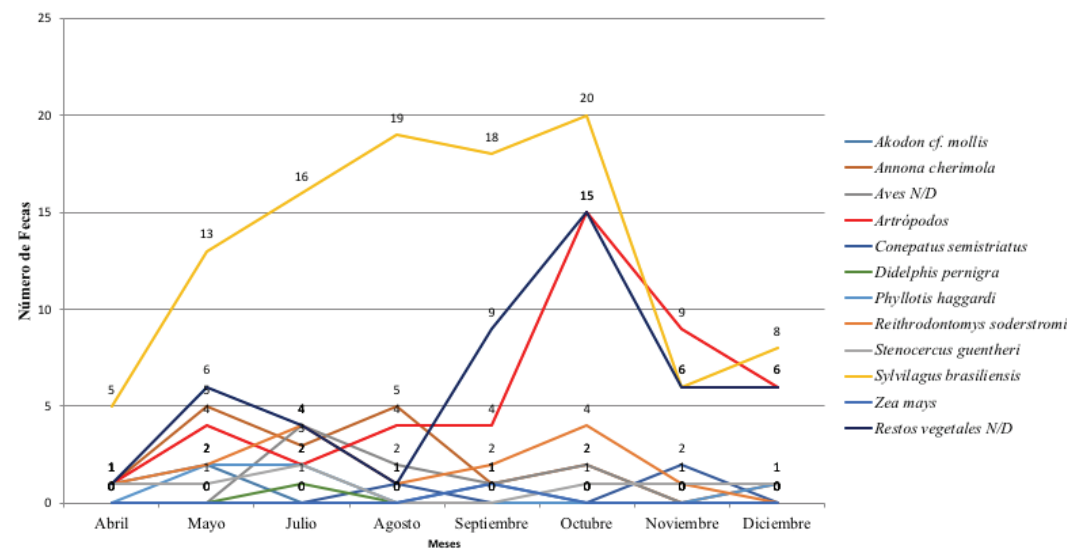

\section{DISCUSIÓN}

\section{Ocurrencia de Presas}

En el caso de los roedores, se ha registrado en ecosistemas de características parecidas al encontrado en Tababela, como lo es el Bosque Protector Jerusalén, donde la dieta estuvo representada mayoritariamente por roedores y lagomorfos [5], de la misma forma la presencia o el aumento en la frecuencia de roedores dentro de la dieta está directamente relacionada a la disponibilidad y variación poblacional de estas presas en el área de estudio [7].

En cuanto al consumo de mamíferos medianos como C. semistiratus y D. pernigra, se puede agregar que estas especies pudieron haber sido consumidas como carroña ya que la presencia de automotores en la zona de estudio era un riesgo que amenazaba a estas especies, así mismo se ha registrado que la presencia de especies como $C$. semistriatus dentro de la dieta es poco ocurrente y además es señal de que las presas recurrentes de $L$. culpaeus en la zona empiezan a escasear, por lo que este cánido se ve obligado a utilizar nuevos recursos para su supervivencia, de la misma manera la baja ocurrencia de marsupiales dentro de la dieta es atribuida a que estas especies son más frecuentes en zonas más cerradas (como arbustales), en donde la presencia de $L$. culpaeus es menor [9].

En relación al consumo de reptiles, se ha atribuido que su consumo está relacionado con la disponibilidad que tienen en el ecosistema, [6], sin embargo, en el área de Tababela, durante las observaciones en campo pudo constatarse una alta disponibilidad de esta especie, bajo este contexto, se podría atribuir la baja incidencia de S. guentheri dentro de la dieta al patrón de actividad de L. culpaeus, el cual en la plataforma es mayoritariamente crepuscular o nocturno, tiempo en el cual S. guentheri no se encuentra activa. 


\section{Variación Mensual en la 0currencia de Presas}

Tras el análisis de la muestras se pudo obtener que S. brasiliensis fue la presa más utilizada por L. culpaeus a lo largo de todos los meses de studio. De la misma forma, se obtuvo que tanto los Restos vegetales No identificados (N/D) y Barotheus andinus, son frecuentemente consumidas por L. culpaeus en el área de la plataforma de Tababela; el mismo caso se presenta para R. soderstromi, que a pesar de que su frecuencia mensual en la dieta fue baja, esta especie estuvo presente en casi todos los meses de muestreo (ver Tabla 1), pudiendo considerarse como una presa recurrente dentro de la dieta de L. culpaeus.

En el caso del resto de ítems alimentarios encontrados, debido a su baja frecuencia dentro de la dieta, pueden ser considerados como capturas esporádicas que L. culpaeus realizó durante sus rondas forrajeo.

De acuerdo con el análisis de frecuencias de ocurrencia de las presas, puede observarse que $S$. brasiliensis fue una especie con alta disponibilidad dentro del área a lo largo del tiempo de estudio, por otra parte, los artrópodos como B. andinus y P. unicolor, fueron especies estacionales, restringidas a los meses de septiembre a noviembre, donde mostraron una mayor disponibilidad debido a su aumento poblacional debido al inicio del periodo reproductivo.

Puede concluirse, que L. culpaeus en la zona de la plataforma es una especie omnívora. Capaz de cambiar su dieta en función de la variación en la disponibilidad de los recursos alimenticios existentes en el medio, lo cual está evidenciado por la aparición de presas esporádicas, sin embargo, el hecho de que existan especies como C. semistriatus dentro de la dieta nos muestra que los recursos comúnmente usados por el lobo de páramo no son suficientes para el mantenimiento de la población de esta especie en el sector.

\section{AGRADECIMIENTOS}

Se agradece al personal del Instituto Nacional de Biodiversidad por el apoyo en la identificación en laboratorio de las presas, en especial a Pablo Moreno por el apoyo en la identificación de los restos de roedores que fueron encontrados y a Jorge Brito por la colaboración en las directrices de la redacción del manuscrito.

\section{CONTRIBUCIÓN DE LOS AUTORES}

Darío Reina redacto el manuscrito y realizó el trabajo de campo. 


\section{REFERENCIAS}

[1] Jiménez, J. E. \& Novaro A. J. (2004). Culpeo, Pseudalopex culpaeus (Molina, 1782) en: “Canids: Foxes, Wolves, Jackals and Dogs: Status Survey and Conservation Action Plan". C. Sillero - Zubiri, M. Hoffmann, D. W. Mcdonald (Eds.), UICN/ SSC Canid Specialist Group. Gland, Suiza y Cambridge, Reino Unido, pp. 44 - 49: carnivoreconservation.org/files/ actionplans/canids.pdf.

[2] Tirira, D. (2007). "Guía de Campo de los Mamíferos del Ecuador", Ediciones Murciélago Blanco. Publicación especial sobre los mamíferos del Ecuador 6. Quito.

[3] Zapata - Ríos, G., Tirira D.G., Armijos J.I. \& Cisneros R. (2011). Lobo de páramo (Lycalopex culpaeus). En: “Libro Rojo de los Mamíferos del Ecuador". D. G. Tirira (Ed.), 2a Edición, Fundación Mamíferos y Conservación, Pontificia Universidad del Ecuador y Ministerio del Ambiente del Ecuador. Publicación Especial sobre los mamíferos del Ecuador 8. Quito, pp. $215-216$.

[4] Medel, R.G. \& Jaksic F.M. (1988). "Ecología de los cánidos sudamericanos: una revisión", Revista Chilena de Historia Natural, 61:67-79: rchn.biologiachile.dl/pdfs/1988/1/Medel_\&_Jaksic_1988.pdf.

[5]Trujillo, F.\&Trujillo,J. (2007). “Alimentación de lobo (Lycalopex culpaeus), en el Bosque Protector Jerusalén, Guayllabamba -Ecuador", Politécnica, 27 (4), Biología, 7: bibdigital.epn.edu.ec/bitstream/15000/3838/1/Alimentaci\%C3\%B3n\%20 del\%20Lobo.pdf.

[6] Guzmán-Sandoval, J., Siefield, W. \& Ferrú, M. (2007). “Dieta de Lycalopex culpaeus (Familia: CANIDAE) en el extremo norte de Chile (Región de Tarapaca)", Gayana, 71(1): scielo.cl/pdf/gayana/v71n1/art01.pdf.

[7] Crespo, J.A. \& De Carlo J.M. (1963). Estudio ecológico de una población de zorros colorados Dusicyon culpaeus culpaeus (Molina) en el oeste de la provincia de Neuquén. Revista del Museo Argentino de Ciencias Naturales "Bernardino Rivadavia"1(1): $1-55$.

[8] Fuentes, E.R. \& Jacksic, F.M. (1979). “Latitudinal size variation of Chilean foxes: Test of alternative hypotheses”, Ecology, 60: jstor.org/stable/1936466

[9] Cornejo - Farfán, A. \& Jiménez -Millón, P. (2001). “Dieta del zorro andino Lycalopex culpaeus (CANIDAE) en el Matorral Desértico del sur del Perú", Revista de Ecología Latinoamericana, 8 (1): cires.org.ve/pdf/recol-v8n1a01.pdf.

[10] Eisengerg, J.F. \& Redford K.H. (1999). "Mammals of the Neotropics, Volume 3: The Central Neotropics", The University of Chicago Press, Chicago.

[11] Castro, S.A., Silva S.I., Meserve P.L., Gutiérrez J.R., Contreras L.C. \& Jaksic, F.M. (1994). “Frugivoría y dispersión de semillas de pimiento (Schinus molle) por el zorro culpeo (Pseudalopex culpaeus) en el Parque Nacional Fray Jorge (IV Región, Chile)", Revista Chilena de Historia Natural, 67: rchn.biologiachile.d/pdfs/1994/2/Castro_et_al_1994.pdf.

[12] Distrito Metropolitano de Quito (DMQ). (2002). Actualización del estudio de Impacto Ambiental de nuevo Aeropuerto de Quito. Informe Final. Quito.

[13] Hammer, Ø., Harper, D.A.T. \& Ryan, P.D. (2001). "PAST: Paleontological statistics software package for education and data analysis", Palaeontologia Electronica 4(1): palaeo-electronica.org/2001_1/past/issue1_01.htm. 